\title{
How should I treat a patient with a mechanical aortic valve prosthesis and recurrent intracranial bleeding on anticoagulation with a patent LIMA to LAD?
}

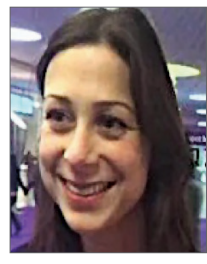

Tiffany Patterson ${ }^{1 *}$, MBBS, BSc, MRCP; William R. Davies², PhD, MRCP, MRCS;

Ronak Rajani², FRCP; Simon Redwood ${ }^{1}$, MD, FRCP, FACC;

Christopher Young'2, MD, FRCS (CTh); Martyn Thomas², MD, FRCP

1. Cardiovascular Division, Rayne Institute, BHF Centre of Research Excellence, King's College London, St Thomas' Hospital, London, United Kingdom; 2. Cardiothoracic Department, St Thomas'Hospital, London, United Kingdom

Invited experts: Johan Bosmans ${ }^{3}$, MD, PhD; Inez Rodrigus ${ }^{4}, M D, P h D ;$ Serguei I. Melnitchouk ${ }^{5}$, MD; Thoralf M. Sundt III , MD

3. Department of Interventional Cardiology, University Hospital Antwerp, Antwerp, Belgium; 4. Department of Cardiac Surgery, University

Hospital Antwerp, Antwerp, Belgium; 5. Division of Cardiac Surgery, Massachusetts General Hospital, Boston, USA

The concluding section "How did I treat?" together with the complete references and the supplementary data are published online at: http://www.pcronline.com/eurointervention/100th_issue/69

\section{CASE SUMMARY}

BACKGROUND: A 62-year-old male underwent mechanical aortic valve replacement and coronary artery bypass grafting. Within 12 months of anticoagulation he developed intracranial haemorrhage requiring neurosurgery. He was referred for consideration of redo aortic valve replacement with a bioprosthesis.

INVESTIGATION: TOE revealed moderate paraprosthetic leak. Diagnostic angiography demonstrated a chronic total occlusion (CTO) of the LAD and patent LIMA supplying a large area of the anterior wall; the graft was retrosternal, in close apposition to the posterior aspect of the manubrium on computerised tomography, suggesting a high risk of damage on redo surgery.

DIAGNOSIS: A patient with a mechanical aortic valve replacement who has suffered recurrent intracranial bleeds and a patent LIMA in close apposition to the manubrium.

TREATMENT: Percutaneous coronary intervention to the LAD CTO followed by a hybrid procedure to secure haemostasis and replace the aortic valve.

KEYWORDS: aortic stenosis, coronary artery disease, Heart Team, redo cardiac surgery

\section{PRESENTATION OF THE CASE}

A 62-year-old male ex-smoker with a background of hypercholesterolaemia and hypertension presented with CCS Class III angina and NYHA Class III breathlessness two years previously. He was found to have significant aortic stenosis in the context of a congenitally bicuspid valve and significant coronary artery disease involving his left anterior descending (LAD) and circumflex arteries. He subsequently underwent mechanical aortic valve replacement and coronary artery bypass grafting with a left internal mammary artery (LIMA) to his LAD and saphenous vein grafts (SVG) to the diagonal and obtuse marginal (OM) vessels. His postoperative recovery was uneventful; however, he did not feel complete resolution of his breathlessness.

Unfortunately, within 12 months of warfarin anticoagulation, he developed the first of three subacute bilateral subdural haemorrhages. For the first two he required neurosurgical intervention (burr hole surgery and drainage) and was left with mild residual neurological deficit which necessitated neuro-rehabilitation. $\mathrm{He}$ raised some concern with regard to his ongoing anticoagulation and the risk of further intracranial bleeding and had considered discontinuing the oral anticoagulation against medical advice. To complicate matters, his symptoms of breathlessness had progressed.

He was referred to our tertiary centre for further investigation and consideration of redo surgery with replacement of his mechanical aortic valve with a bioprosthesis to allow cessation of anticoagulation. A transoesophageal echocardiogram (Figure 1, Moving image 1) revealed a moderate paraprosthetic leak localised to the non-coronary cusp region of the mechanical aortic valve with preserved left ventricular function. Diagnostic coronary and graft

\footnotetext{
*Corresponding author: Cardiovascular Division, Rayne Institute, BHF Centre of Research Excellence, King's College London,
} St Thomas'Hospital, Westminster Bridge Road, London, SE1 7EH, United Kingdom. E-mail: tiffanypatterson05@gmail.com 
angiography (Figure 2, Moving image 2) demonstrated a chronic total occlusion (CTO) of the LAD with a patent LIMA supplying a large area of the anterior wall, an occluded SVG to diagonal, significant proximal disease in the circumflex artery with occluded SVG to OM and an unobstructed right coronary artery. A tortuous route of the LIMA graft was also noted that crossed the midline of the sternum to the right side of the chest. An ECG-gated coronary computed tomographic (CT) angiographic study with 3D

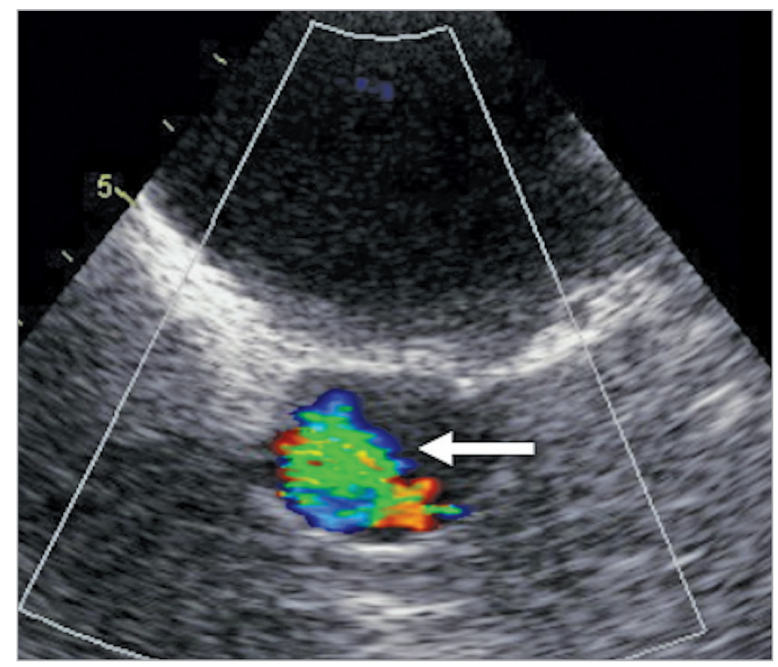

Figure 1. Transoesophageal echocardiogram short-axis view of the aortic valve with moderate paraprosthetic jet of aortic regurgitation (arrow). reconstruction (Figure 3, Moving image 3) confirmed the retrosternal nature of the LIMA graft and also showed a close apposition of the graft to the posterior surface of the manubrium.

This suggested a high risk of damage to the patent LIMA if the patient were to undergo redo surgery. The risks were twofold, first ischaemia and infarction to the anterior wall and, second, the risk of uncontrollable haemorrhage prior to the initiation of cardiopulmonary bypass should the LIMA be disrupted.

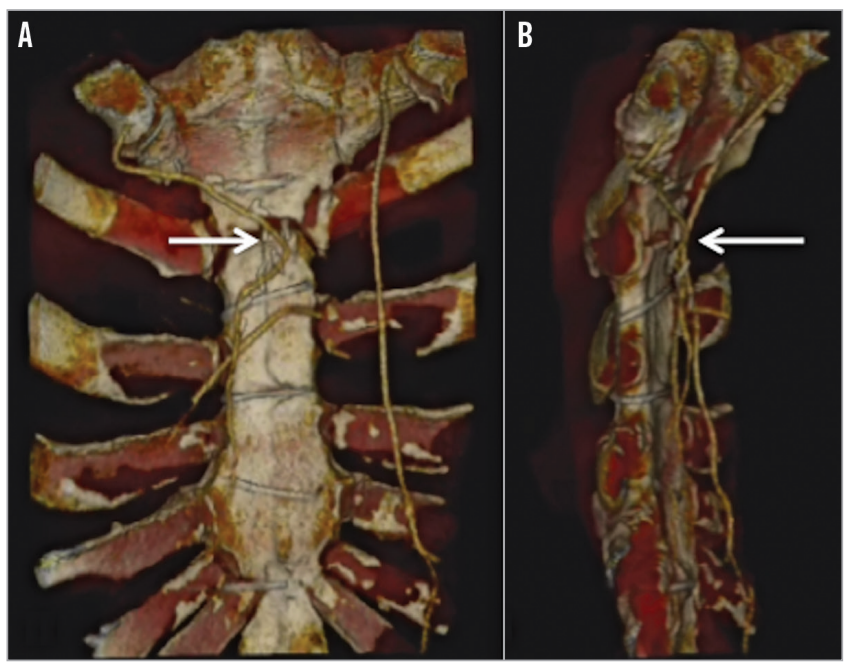

Figure 3. CT coronary angiogram. $3 D$ reconstruction of LIMA traversing the midline $(A)$ and in close proximity to the sternum (B).

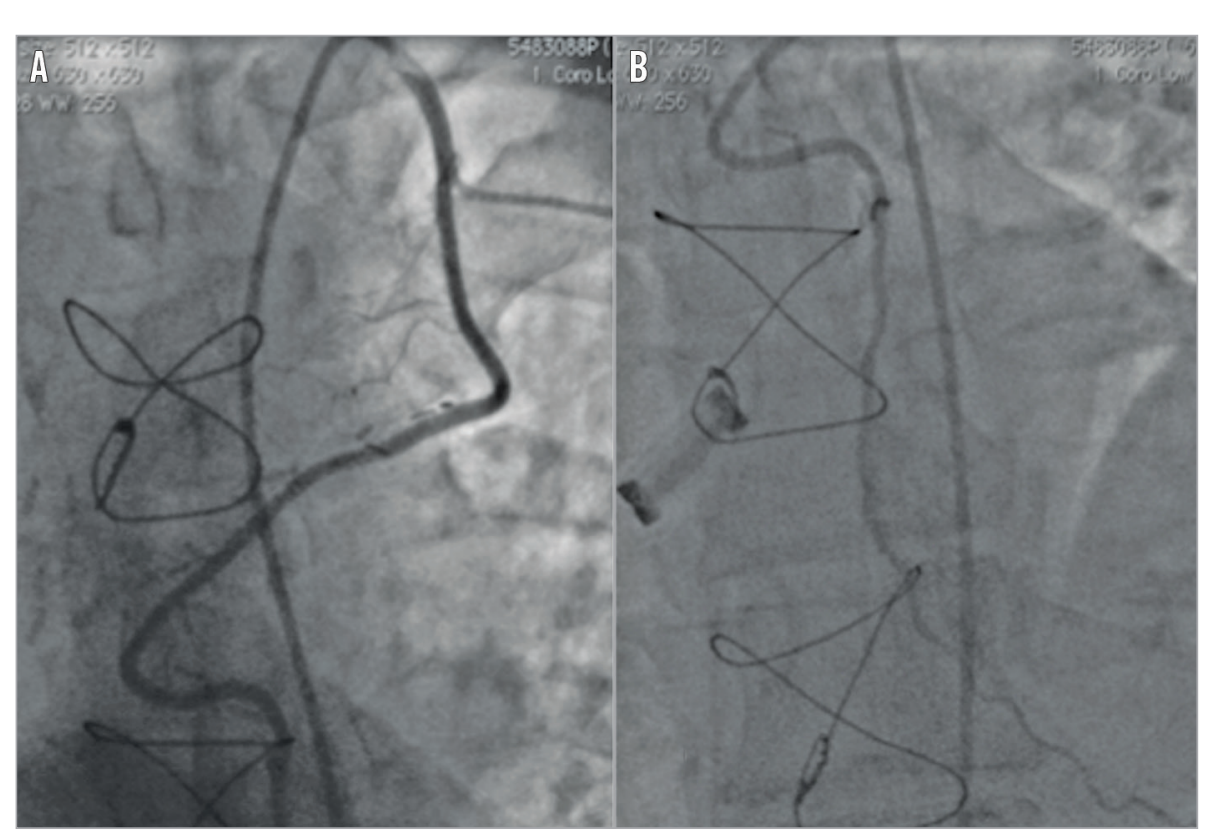

Figure 2. Diagnostic coronary angiogram and graft study. Patent LIMA to LAD following a tortuous course traversing the midline of the chest (A) with good distal run-off (B). 


\section{How would I treat? THE INVITED EXPERTS' OPINION}

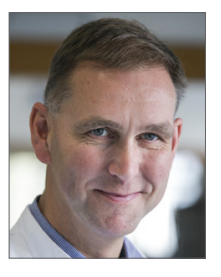

Johan Bosmans ${ }^{3 *}, \mathrm{MD}, \mathrm{PhD}$; Inez Rodrigus ${ }^{4}, \mathrm{MD}, \mathrm{PhD}$

3. Department of Interventional Cardiology, University Hospital Antwerp, Antwerp, Belgium; 4. Department of

Cardiac Surgery, University Hospital Antwerp, Antwerp, Belgium

In our opinion, based on the multidisciplinary Heart Team discussion, the most important and threatening clinical problem of this patient is the recurrent intracranial haemorrhages within 12 months of warfarin anticoagulation, after placement of a mechanical aortic valve prosthesis (AVR). The symptoms of breathlessness certainly might be related to the paraprosthetic aortic valve leakage. However, with transoesophageal evaluation, this leakage is only moderate with preserved left ventricular function, and therefore it does not warrant urgent treatment. The saphenous vein graft to the obtuse marginal vessels is occluded, with significant disease in the circumflex artery. However, because of the absence of angina and the relatively diffuse atherosclerotic disease, and also in order to avoid even more aggressive additional antiplatelet therapy after eventual coronary stenting, a conservative strategy can be defended for this specific problem.

In our treatment strategy for this patient with clearly increased surgical risk, we should first start with adapting warfarin anticoagulant strategy by lowering INR targets (1.5-2.1), in combination with frequent (self-)monitoring (once or twice a week). Indeed, there has been a gradual trend towards lower INR targets for mechanical prostheses to minimise the risk of anticoagulation-related bleeding. The LOWERing the INtensity of oral anticoaGulant Therapy in patients with mechanical aortic valve replacement trial ${ }^{1}$ randomised patients with mechanical AVR to low-dose (INR 1.5-2.5) versus standard dose (INR 2-3) anticoagulation. After 2,198 patient-years, low-dose anticoagulation was associated with no difference in thromboembolic events but significant reduction of bleeding events. In a study ${ }^{2}$ of patients with mechanical heart valves randomised to low-dose INR self-control (target AVR 1.8-2.8) or very low-dose INR selfcontrol once or twice a week (target AVR 1.6-2.1), two-year freedom from bleeding was significantly lower in the very low-dose group with no differences in thrombotic events.

The potential use of non-warfarin oral anticoagulants (NOACs) as alternatives is being studied. However, there is currently no role for the NOACs for thromboembolic prophylaxis for valvular prostheses, mainly based on the dabigatran trial ${ }^{3}$, but trials with rivaroxiban are ongoing.

If, despite adapted warfarin therapy, new intracranial haemorrhage should occur, surgical treatment becomes unavoidable. In case of redo surgery a crucial part is to perform safe resternotomy in order to avoid damage of the patent LIMA. Groin vessels can be prepared for cardiopulmonary bypass and RIMA or A radialis harvested for bypassing the circumflex artery and obtuse marginal vessels, using retrograde cardioplegia. Finally, an aortic valve bioprosthesis will be inserted. Afterwards, the patient will be treated with aspirin (50-100 mg/d).

\section{Conflict of interest statement}

The authors have no conflicts of interest to declare. 


\section{How would I treat? THE INVITED EXPERTS' OPINION}

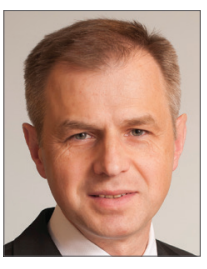

Serguei I. Melnitchouk ${ }^{5 *}$, MD; Thoralf M. Sundt III' ${ }^{5}$ MD

5. Division of Cardiac Surgery, Massachusetts General Hospital, Boston, MA, USA

The case presented is a challenging one indeed. We agree that, with recurrent intracranial bleeding on anticoagulation as well as a moderate paraprosthetic leak, there is an indication for reoperation. Normally in such instances one would plan to perform a repeat sternotomy, revise the bypass graft to obtuse marginal branch, and replace the mechanical valve with a bioprosthesis. The patent ITA appears to be behind but not adherent to the sternum and one can safely perform resternotomy. Accordingly, we would consider addressing the circumflex lesion by a percutaneous coronary intervention beforehand to make the operation simpler and so that injury, if it were to occur, would be less catastrophic. The operation itself can be done either via resternotomy or right anterior minithoracotomy.

As for the former, we can reduce the risk of reopening by initiating cardiopulmonary bypass (CPB) via peripheral cannulation prior to the division of the posterior table of the sternum. Cardiac decompression achieved with CPB allows the cardiac structures to fall away from the sternum and facilitates safer dissection. Furthermore, moderate hypothermia $\left(32^{\circ} \mathrm{C}\right)$ would provide additional protection if an inadvertent injury to the LIMA did occur and would give us a bit more time for primary repair of the LIMA or placement of an additional venous graft ${ }^{4}$.

There is no need to isolate the ITA, although in this case one would probably need to mobilise it simply to access the aorta for valve replacement. It has been shown by multiple authors that administration of retrograde cardioplegia, while leaving a patent ITA unclamped, provides an adequate myocardial protection ${ }^{5,6}$. It is our practice to do so with moderate hypothermia $\left(32^{\circ} \mathrm{C}\right)$ in all such cases. Given a likely left ventricular hypertrophy, we would also give additional cardioplegia antegradely once the root is open. One can then explant the old mechanical prosthesis and place a new bioprosthesis in a standard fashion.

An alternative approach would involve a right anterior minithoracotomy and peripheral bypass. If applicable, utilisation of sutureless valve technology would help tremendously with an expedient valve placement in this situation. However, this approach should be carefully weighed against the risk of injuring the LIMA graft in the setting of minithoracotomy.

\section{Conflict of interest statement}

The authors have no conflicts of interest to declare. 


\section{How did I treat?}

\section{ACTUAL TREATMENT AND MANAGEMENT OF THE CASE}

The Heart Team considered the case for redo aortic valve replacement and coronary artery bypass grafting. An agreement was reached that bioprosthetic valve replacement would be beneficial; however, the unusually tortuous course and close apposition of the mammary to the posterior aspect of the sternum gave significant cause for concern. A careful plan was made to address the risk of both infarction and bleeding using a stepwise approach.

\section{Procedure 1}

The patient was first to undergo coronary intervention with PCI to the CTO of the LAD using a bare metal stent strategy necessitating only one month of dual antiplatelet therapy, to form a new conduit to perfuse the anterior wall.
6 Fr right femoral arterial access was obtained, $70 \mathrm{IU} / \mathrm{kg}$ heparin administered and a Judkins Left 4 (Medtronic, Minneapolis, MN, USA) guide catheter was used to introduce a PT Graphix ${ }^{\mathrm{TM}}$ Intermediate Guide Wire (Boston Scientific, Marlborough, MA, USA) to the distal native LAD supported by a 1.25 Ryujin $^{\mathrm{TM}}$ (Terumo Europe NV, Leuven, Belgium) over-the-wire (OTW) balloon. The lesion was predilated with the 1.25 OTW balloon followed by further lesion preparation with a $2 \times 12 \mathrm{~mm}$ compliant balloon. Two overlapping stents were successfully deployed in the mid $\left(3 \times 28 \mathrm{~mm}\right.$ OMEGA ${ }^{\mathrm{TM}}$; Boston Scientific) and proximal $(3 \times 20 \mathrm{~mm}$ OMEGA) LAD with a further stent distally $(2.5 \times 16 \mathrm{~mm}$ OMEGA) with excellent angiographic result and TIMI 3 flow (Figure 4, Moving image 4A-Moving image 4D).

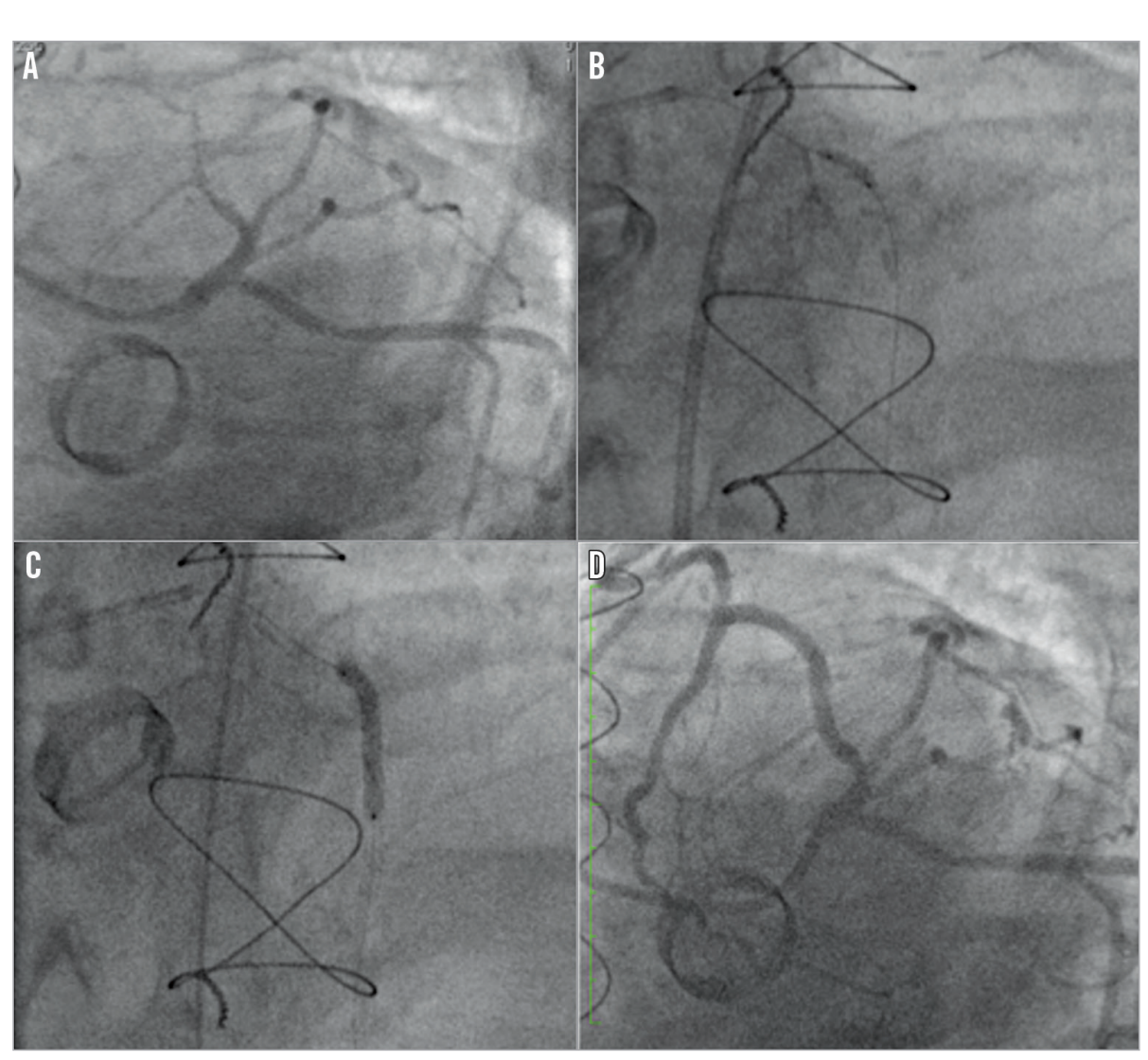

Figure 4. PCI to CTO LAD. The severely diseased LAD (A) was predilated (B) and stented $(C)$ with excellent final angiographic result and TIMI 3 flow (D) in a large vessel. 


\section{Procedure 2}

Six weeks later the patient underwent redo AVR and bypass grafting in the hybrid cardiology/cardiac surgery theatre with the intention of utilising integrated interventional cardiology techniques for balloon occlusion of the LIMA prior to sternotomy.

Femoral arterial access was obtained and a 6 Fr Launcher ${ }^{\circledR}$ IMA guide catheter (Medtronic) was used to intubate the LIMA and introduce two wires into the LIMA to LAD. Thereafter, two $3.0 \mathrm{~mm}$ semi-compliant balloons were placed in the IMA, one providing proximal occlusion and one distal (Figure 5, Moving image 5). Trial proximal balloon inflation caused no electrocardiographic evidence of ischaemia. The patient was systemically heparinised and the two angioplasty balloons inflated. The resternotomy was initially uneventful with the successful removal of the sternal wires and access was gained into the thorax using an oscillating saw. However, on sternal retraction, the internal mammary artery was stretched and transected at the junction of the proximal and distal third. The two angiography wires were clearly visible at the site of transection. Despite recoil of the distal portion of the artery, no haemorrhage was evident. By tracking of the angioplasty wires, the proximal and distal ends of the LIMA were identified

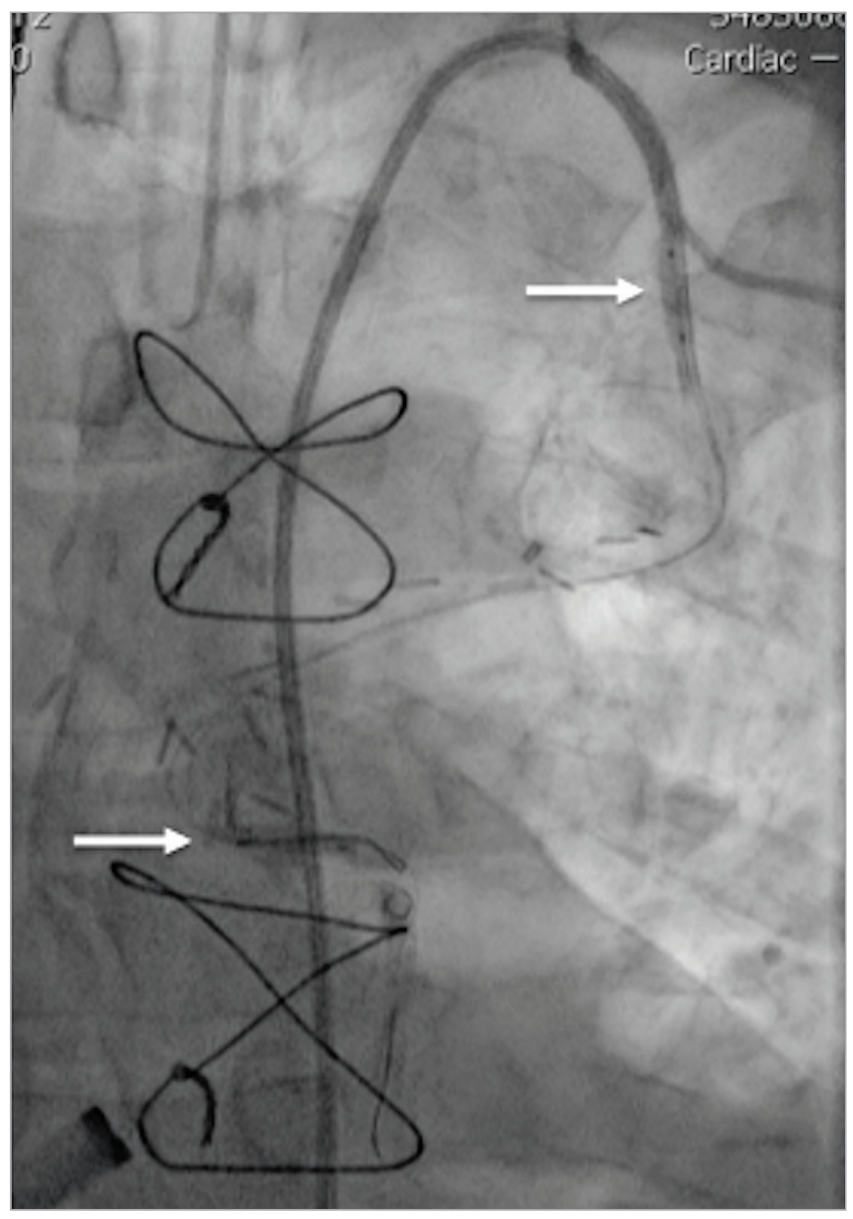

Figure 5. Hybrid procedure. Intubation of LIMA with IM guide catheter and two wires introduced to vessel with balloons inflated proximally and distally (arrows), successfully occluding flow. and oversewn following removal of the angioplasty wires and the balloons ligated. The electrocardiogram remained isoelectric throughout, and there were no signs of haemodynamic compromise. The heart was then carefully mobilised and cannulated for cardiopulmonary bypass. The mechanical valve was excised and a $23 \mathrm{~mm}$ bioprosthetic Carpentier-Edwards Magna Ease (Edwards Lifesciences, Irvine, CA, USA) was implanted with anastomosis of SVG to OM.

The patient made an uneventful postoperative recovery and was discharged home on day 4. A pre-discharge echocardiogram showed a well-seated bioprosthesis, with no evidence of anterior wall systolic compromise.

\section{Conclusion}

In a patient requiring resternotomy in the presence of a patent left internal mammary graft, we present a novel approach to mitigate the potential pitfalls of injury to the graft utilising integrated interventional cardiology techniques in the hybrid theatre. The case also demonstrates the need for integrated working within a dedicated Heart Team for complex cases that draws upon the specific skills of interventional and imaging cardiologists along with cardiothoracic surgical colleagues.

\section{Conflict of interest statement}

The authors have no conflicts of interest to declare.

\section{References}

1. Torella M, Torella D, Chiodini P, Franciulli M, Romano G, De Santo L, De Feo M, Amarelli C, Sasso FC, Salvatore T, Ellison GM, Indolfi C, Cotrufo M, Nappi G. LOWERing the INtensity of oral anticoaGulant Therapy in patients with bileaflet mechanical aortic valve replacement: results from the LOWERING-IT trial. Am Heart J. 2010;160:171-8.

2. Koertke H, Zittermann A, Wagner O, Ennker J, Saggau W, Sack FU, Cremer J, Huth C, Braccio M, Musumeci F, Koerfer R. Efficacy and safety of very low-dose self-management of oral anticoagulation in patients with mechanical heart valve replacement. Ann Thorac Surg. 2010;90:1487-93.

3. Eikelboom JW, Connolly SJ, Brueckmann M, Granger CB, Kappetein AP, Mack MJ, Blatchford J, Devenny K, Friedman J, Guiver K, Harper R, Khder Y, Lobmeyer MT, Maas H, Voigt JU, Simoons ML, Van de Werf F; RE-ALIGN Investigators. Dabigatran versus warfarin in patients with mechanical heart valves. $N$ Engl J Med. 2013;369:1206-14.

4. Gillinov AM, Casselman FP, Lytle BW, Blackstone EH, Parsons EM, Loop FD, Cosgrove DM 3rd. Injury to a patent left internal thoracic artery graft at coronary reoperation. Ann Thorac Surg. 1999;67:382-6.

5. Park CB, Suri RM, Burkhart HM, Greason KL, Dearani JA, Schaff HV, Sundt TM 3rd. What is the optimal myocardial preservation strategy at re-operation for aortic valve replacement in the presence of a patent internal thoracic artery? Eur J Cardiothorac Surg. 2011;39:861-5. 
6. Byrne JG, Karavas AN, Filsoufi F, Mihaljevic T, Aklog L, Adams DH, Cohn LH, Aranki SF. Aortic valve surgery after previous coronary artery bypass grafting with functioning internal mammary artery grafts. Ann Thorac Surg. 2007;73: $779-84$.

\section{Supplementary data}

Moving image 1. Transoesophageal echocardiogram. Short-axis view of the aortic valve with moderate paraprosthetic jet of aortic regurgitation.

Moving image 2. Diagnostic coronary angiogram and graft study. Patent LIMA to LAD following a tortuous course traversing the midline of the chest with good distal run-off.

Moving image 3. CT coronary angiogram. 3D reconstruction of LIMA traversing the midline and in close proximity to the sternum.
Moving image 4A. PCI to CTO LAD. LAO caudal view of proximal left main stem and chronically occluded LAD.

Moving image 4B. PCI to CTO LAD. PT Graphix intermediate wire (Boston Scientific) to the distal native LAD supported by a 1.25 Ryujin (Terumo) over-the-wire (OTW) balloon. Predilated with the 1.25 OTW balloon followed by further lesion preparation with a $2 \times 12 \mathrm{~mm}$ compliant balloon.

Moving image 4C. PCI to CTO LAD. Two overlapping stents deployed in the mid $(3 \times 28 \mathrm{~mm}$ OMEGA; Boston Scientific) and proximal $(3 \times 20 \mathrm{~mm}$ OMEGA) LAD with a further stent distally $(2.5 \times 16$ mm OMEGA)

Moving image 4D. PCI to CTO LAD. Excellent final angiographic result with TIMI 3 flow.

Moving image 5. Hybrid procedure. Intubation of LIMA with IM guide catheter and two wires introduced to vessel with balloons inflated proximally and distally, successfully occluding flow. 